\title{
Analysis of the optic-type collective mode in liquid selenium to determine the local structure
}

\author{
Ayano Chiba, ${ }_{1,3}^{13}$ Yoshinori Ohmasa, ${ }^{2}$ Stephen M. Bennington, ${ }^{3}$ Jon W. Taylor, ${ }^{3}$ and Makoto Yao $^{2}$ \\ ${ }^{1}$ Department of Physics, Keio University, Yokohama 223-8522, Japan \\ ${ }^{2}$ Department of Physics, Graduate School of Science, Kyoto University, Kyoto 606-8502, Japan \\ ${ }^{3}$ Rutherford Appleton Laboratory, Chilton, Didcot OX11 OQX, United Kingdom
}

(Received 4 March 2008; published 10 April 2008)

\begin{abstract}
We have performed inelastic neutron scattering measurements for liquid selenium to investigate the dynamic structure, $S(Q, E)$, of the chainlike molecules in the liquid state. We observed a distinct optic-type mode at around $31 \mathrm{meV}$, whose $Q$ dependence of the intensity, $S(Q, E \sim 31 \mathrm{meV})$, was found to be reproduced by a simple approximation of "three-body" model. We propose the possibility of extracting structural parameters such as bond length and angle by constant $E$ spectra by the use of this simple model, which at the same time provides us a clearcut physical meaning of the $Q$ dependences.
\end{abstract}

DOI: 10.1103/PhysRevB.77.132202

PACS number(s): 61.25.Em, 61.05.F-, 61.20.-p

Measuring the static structure factor, $S(Q)$, of liquids is a powerful and the most common method to determine the static local structure of liquids. However, $S(Q)$ does not retain information on three-body or many-body correlations. In contrast, the dynamic structure factor, $S(Q, E)$, includes the correlated atomic displacements for each $E$, and thus, manybody correlations can be extracted for a certain vibrational mode at $E$ if the mode is simple enough. In this Brief Report, we propose the possibility of extracting structures such as bond length and angle in liquids from constant- $E$ spectra of inelastic neutron scattering (INS). The point here is the physically clearcut and simple model employed, by which one can see the physical origin of the apparently complex $Q$ dependence of optic-type mode in liquid. This method is fundamentally different from extended x-ray absorption fine structure (EXAFS) in that this model works in a physically simple framework (e.g., Born approximation, as noted below) and intrinsically based on dynamics.

In the large-scale and low-frequency limit, $S(Q, E)$ for liquids will be governed by hydrodynamics; e.g., $D Q^{2}$ law, sound velocity, etc. On the other hand, in the highermomentum region, the dynamic structure will show "solidlike" character, such as the minimum of the acoustic dispersion curve that resembles umklapp scattering in crystals. ${ }^{1}$ In the higher-energy region, $S(Q, E)$ for liquids can have optictype vibrational contributions if the system is ionically ${ }^{2}$ or covalently ${ }^{3}$ bonded. In molecular liquids, intramolecular motions dominate this region, in contrast to the lower- $Q-E$ region where intermolecular motions and couplings play important roles.

To clarify the optic-type atomic motions in disordered systems, several attempts have so far been made to estimate and interpret the $Q$ dependence of $S(Q, E)$ at a given $E$. In the initial INS studies of Carpenter and Price, ${ }^{4}$ e.g., such constant- $E$ spectra for optic modes are investigated for vitreous $\mathrm{SiO}_{2}$. Considerable number of studies followed for optic modes in amorphous solids. ${ }^{5}$ For liquids, we can cite INS studies on water, ${ }^{6}$ liquid $\mathrm{Ga},{ }^{7}$ expanded rubidium, ${ }^{8}$ liquid $\mathrm{DF},{ }^{3}$ molten $\mathrm{Li}_{30} \mathrm{Bi}_{70},{ }^{9}$ etc. We may also cite theoretical approach which shows a condition of existence for optic mode in binary liquid. ${ }^{10}$ However, it has been difficult to quantitatively perform accurate and clearcut interpretation of experimental constant- $E$ spectra, as a result of the complex struc- tures for liquids and even for amorphous solids. Here, we show the spectra can be well reproduced by simple models, and furthermore, quantitative structures can be extracted by the model. To demonstrate the validity of our analysis, Se was chosen because of following reasons: (1) Se is very favorable to study the collective excitations by neutron scattering because of its high coherency, i.e., $\sigma_{c} / \sigma_{s}=96.1 \%,{ }^{11}$ where $\sigma_{c}$ and $\sigma_{s}$ denote the coherent and total neutron scattering cross section, respectively. (2) $l$-Se offers the possibility of quantitative investigations by simple models because of its chainlike structure with a low coordination number, two, and the fact that its static and dynamic structure have already been extensively studied. ${ }^{12}$ (3) The stretching mode can be clearly distinguished from other modes for $l$-Se and is expected to preserve in wide $Q$ range because of the covalent bond. (4) The stretching mode is located in the energy region, which is high enough to neglect the $Q$ dependence of the main features of quasielastic contribution, which resembles the $Q$ dependence of $S(Q)$. Thus, we shall focus on the constant- $E$ spectra for the stretching mode in this Brief Report because the $Q$ dependence is supposed to be explained by the atomic displacements of the stretching mode without other contributions.

The INS experiments were performed on the MARI direct geometry, time of flight spectrometer at ISIS, and Rutherford Appleton Laboratory in the United Kingdom. We have carried out measurements for $l$-Se at slightly above the melting point, $237{ }^{\circ} \mathrm{C}$, with an incident neutron energy $E_{i}=50 \mathrm{meV}$. The elastic resolution was about $0.8 \mathrm{meV}$ half-width at half maximum, estimated from the vanadium-standard-sample run. Because the sample is corrosive in the liquid state, it was sealed in a fused quartz tube. Further details of the experiments, data analyses and corrections are described in Ref. 13.

By the use of the Born approximation, the $Q$ dependence of the neutron cross section is governed by $S(Q, E)$ $=(2 \pi \hbar N)^{-1} \int d t e^{-i E t / \hbar} \Sigma_{l, l^{\prime}}\left\langle e^{-i \boldsymbol{Q} \cdot\left[\boldsymbol{m}_{l}+\boldsymbol{u}_{l}(0)\right]} e^{i \boldsymbol{Q} \cdot\left[\boldsymbol{m}_{l^{\prime}}+\boldsymbol{u}_{l^{\prime}}(t)\right]}\right\rangle$, where $N$ is the total number of atoms, $\boldsymbol{m}_{l}$ is the position vector, and $\boldsymbol{u}_{l}$ is the atomic displacement of the $l$ th nucleus, and the angular brackets denote the thermal average. Thus, the $Q$ dependence of the one-phonon term is calculated by use of Bloch's identity and Baker-Campbell-Hausdorff $(\mathrm{BCH})$ formula and is 


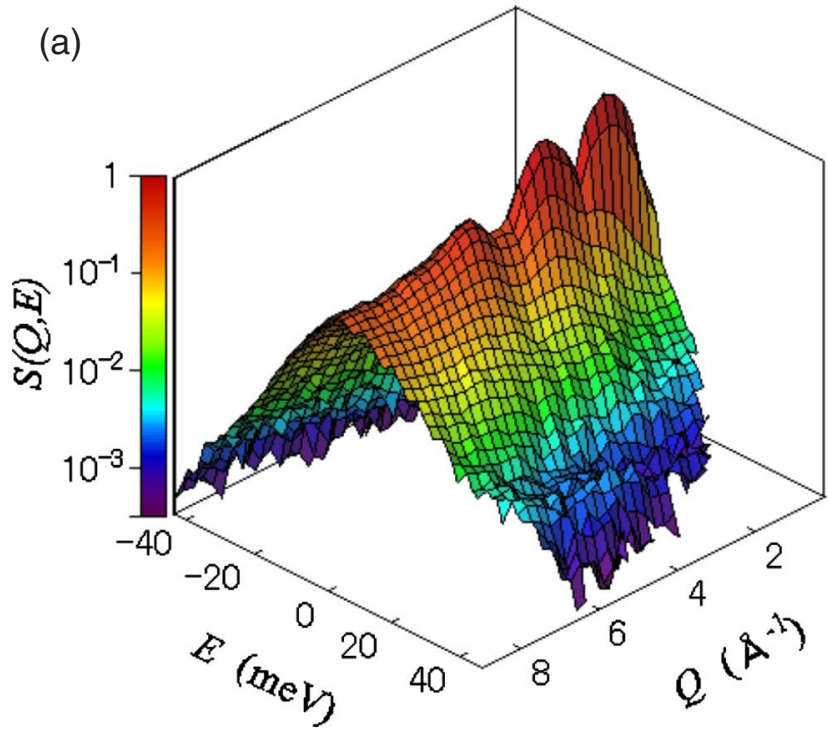

(b)

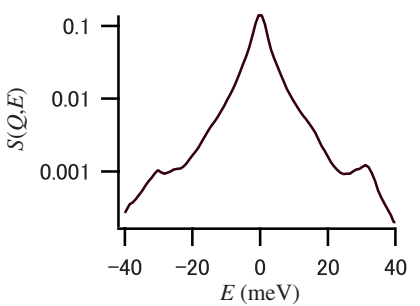

FIG. 1. (a) (Color online) Two-dimensional intensity map of $S(Q, E)$ for $l$-Se at $237^{\circ} \mathrm{C}$. (b) Constant- $Q$ spectra of (a) at $Q=4 \AA^{-1}$.

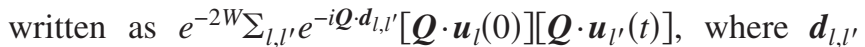
is the internuclear distance vectors and $2 W=\left\langle(\boldsymbol{Q} \cdot \boldsymbol{u})^{2}\right\rangle$ is the Debye-Waller factor. Here, we neglected the higher-order terms in the BCH series, such as $-\frac{1}{12}\left(\left\{\left[\boldsymbol{m}_{l}+\boldsymbol{u}_{l}(0)\right],\left[\boldsymbol{m}_{l^{\prime}}\right.\right.\right.$ $\left.\left.\left.+\boldsymbol{u}_{l^{\prime}}(t)\right]\right\},\left[\boldsymbol{m}_{l}+\boldsymbol{u}_{l}(0)\right]\right)+\frac{1}{12}\left(\left\{\left[\boldsymbol{m}_{l}+\boldsymbol{u}_{l}(0)\right],\left[\boldsymbol{m}_{l^{\prime}}+\boldsymbol{u}_{l^{\prime}}(t)\right]\right\},\left[\boldsymbol{m}_{l^{\prime}}\right.\right.$ $\left.+\boldsymbol{u}_{l^{\prime}}(t)\right]$ ) and others that involves more commutation relations, where the braces and thick round brackets denote the commutator. Since the system is isotropic, we take the orientational average of the one-phonon term, which gives ${ }^{14}$

$$
\begin{aligned}
I^{E}(Q)= & e^{-2 W} Q^{2} \sum_{l, l^{\prime}}\left[\frac{1}{3}\left(\boldsymbol{u}_{l} \cdot \boldsymbol{u}_{l^{\prime}}\right) j_{0}\left(Q d_{l, l^{\prime}}\right)+\left(\frac{1}{3}\left(\boldsymbol{u}_{l} \cdot \boldsymbol{u}_{l^{\prime}}\right)\right.\right. \\
& \left.\left.-\frac{1}{d_{l, l^{\prime}}^{2}}\left(\boldsymbol{d}_{l, l^{\prime}} \cdot \boldsymbol{u}_{l}\right)\left(\boldsymbol{d}_{l, l^{\prime}} \cdot \boldsymbol{u}_{l^{\prime}}\right)\right) j_{2}\left(Q d_{l, l^{\prime}}\right)\right],
\end{aligned}
$$

where $I^{E}(Q)$ denotes the $Q$ dependence of $S(Q, E)$ at certain $E$, and $j_{0}(x)$ and $j_{2}(x)$ are the spherical Bessel functions.

To analyze the experimental $S(Q, E=$ const $)$, calculating the dynamic radial distribution function, $g(r, E)$, is one method. ${ }^{5}$ However, the Fourier transformation of the limited $Q$ range which varies for each $E$ will cause experimental error in $g(r, E)$. Fitting Eq. (1) in $Q$ space, on the other hand, is simple and direct.

Figure 1(a) shows a two-dimensional intensity map of the measured $S(Q, E)$ for $l$-Se at $237^{\circ} \mathrm{C}$ and Fig. 1(b) shows the spectrum of $S\left(Q \sim 4 \AA^{-1}, E\right)$. One can see in (b) clear modes around $\pm 31 \mathrm{meV}$ and weak shoulders around $\pm 15 \mathrm{meV}$. The mode at $31 \mathrm{meV}$ is assigned to the stretching mode, and that at $15 \mathrm{meV}$ to the bending mode. ${ }^{12}$ As we have stated in Ref. 13, the stretching mode for $l$-Se show remarkable shift to higher $E$ than that for solid trigonal $(t-) \mathrm{Se}$. We note that the mode lies around $28 \mathrm{meV}$ for $t$-Se. ${ }^{15}$ This shift is due to the weaker intermolecular correlations in the liquid state than in the trigonal state because the reduced intermolecular coupling leads to increased intramolecular bonding in this system. ${ }^{16}$ Thus, we approximate to regard the dynamic structure of a Se molecule in the liquid state as that similar to the isolated Se chain, when one examines the $Q$-dependence of $S(Q, E)$ at the energy of the stretching mode.

Figures 2(b) and 2(c) show the constant- $E$ spectra extracted from the energy region of the stretching mode, and Fig. 2(a) shows the static structure factor, $S(Q)$, derived by the integration over the accessible $E$ range. One sees that the $Q$ dependence of the stretching mode is completely different from that of $S(Q)$. To clarify what kind of the atomic movement causes the maximum at $4.7 \AA^{-1}$ and the minimum at $5.6 \AA^{-1}$ in Fig. 2(b), we adopted the motions of isolated Se molecule.

First, we tested two-body correlations as a first approximation, assuming the simplest model for $\mathrm{Se}_{2}$ molecular gas, because the mode under consideration is the bond-stretching mode. The solid line in Fig. 2(b) shows the best fit of this two-body model to the experimental data. We used the modified form of Eq. (1) for the out-of-phase motions of pairs of atoms. The dotted line shows the self-scattering term, which monotonously increases as $Q^{2}$. We note that the fitting procedures and the provisional results have been presented in Ref. 17. We found that the prominent shoulder around $4.7 \AA^{-1}$ can be roughly reproduced by this model, although the model is naive compared to the real Se molecules composed of many atoms. The fitting result, $r=2.36 \AA$, was found to be in fairly good agreement with the value $r$ $=2.34 \AA$ obtained by $\mathrm{x}$-ray diffraction and EXAFS measurements, ${ }^{18}$ where $r$ denotes the bond length [see Fig. 3(b)]. However, the minimum at $5.6 \AA^{-1}$ as well as clear maximum at $4.7 \AA^{-1}$ cannot be reproduced by the model, with only short range order.

We therefore extended the model to deal with three-body correlations, assuming that the stretching mode for $l$-Se resembles that for $t$-Se. We adopted the $A_{1}$ mode that is the most high-energy vibrational mode for the trigonal phase. As shown in Fig. 3(a), the $A_{1}$ mode is the chain expansion mode in which each atom moves in the basal plane. For the firstnearest neighbor in the $A_{1}$ mode, Eq. (1) can be reduced to $I_{\text {distinct }}^{E}(Q) \propto Q^{2}\left[-\frac{1}{6} j_{0}(r Q)+\frac{7}{12} j_{2}(r Q)\right]$. For the second-nearest 


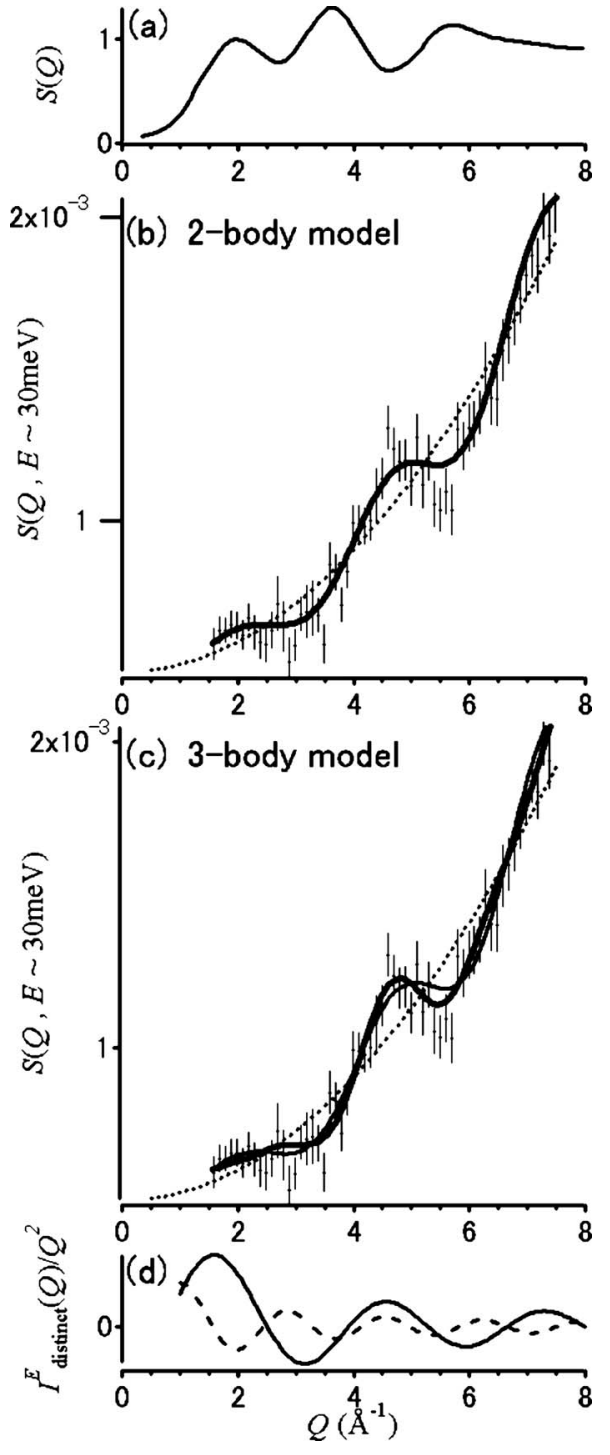

FIG. 2. (a) $S(Q)$ for $l$-Se at $237^{\circ} \mathrm{C}$ obtained by integration over $E$. (b) Constant $E$ spectra at $E=31 \mathrm{meV}$. The closed circles with error bars indicate the experimental data. The solid line shows a fit of "two-body" model based on the motions of stretching mode of $\mathrm{Se}_{2}$ molecules. The dotted line shows the self-scattering term. (c) The same data as (b) with a fit of "three-mody" model shown by thick line. This model gives the motions of first- and second-nearest neighbors in a chain which is shown in Fig. 3. The thin line shows the first-neighbor contribution to the thick line. (d) The solid and dashed lines show the purely distinct part of the first- and secondnearest neighbor contributions, respectively, to the fitted thick line in (c).

neighbor in the same molecule, this equation also applies if $r$ is replaced by the distance between an atom and its secondnearest neighbor in the chain $r_{2}$ [see Fig. 3(b)] because three atoms in Fig. 3(a) symmetrically moves in the case of $A_{1}$ mode. This three-body method produces a better fit to the data, both for the minimum at $5.6 \AA^{-1}$ and the peak at $4.7 \AA^{-1}$. The contributions of the purely distinct term of twoand three-body interactions are shown in Fig. 2(d). One sees that the prominent shoulder of the experimental data at $4.7 \AA^{-1}$ is due to that the solid and dashed lines in Fig. 2(c) (a)

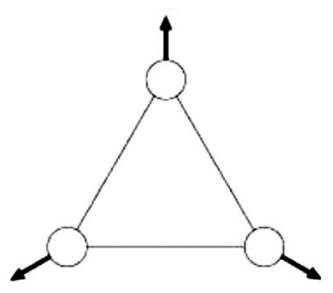

(b)

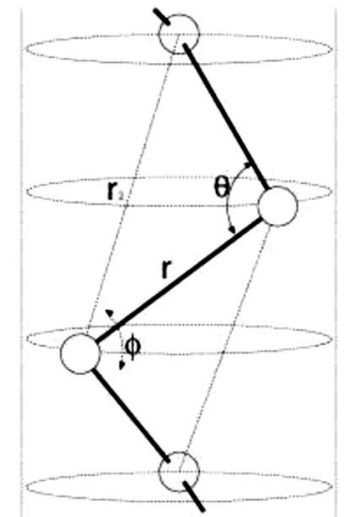

FIG. 3. (a) Top view of the structure of $t$-Se with a displacement pattern for the $A_{1}$ mode. (b) Side view of that with internal coordinates.

are in phase at around $4.7 \AA^{-1}$ with each other. That is, the constant- $E$ structure factor for the two-body correlation and that for three-body one are in phase, whereas these two contributions are basically out-of-phase at other $Q$. One also can see that the minimum at $5.6 \AA^{-1}$ is caused by the three-body correlations.

In addition to the better fit of this three-body model than that of the two-body one, the fitting result, $r=2.34 \pm 0.04 \AA$, shows an excellent agreement with the value of $2.34 \AA$ by earlier experiments. We should, however, note that the difference between $r$ obtained by use of this three-body and the former two-body model is rather smaller than the resolution. The bond angle obtained at the same time, $\theta=107^{\circ}$ is also in good agreement with the values derived from neutron-scattering, ${ }^{19} \mathrm{x}$-ray diffraction, ${ }^{20}$ and $a b$ initio MD simulations ${ }^{21,22}$ that concluded $\theta=103^{\circ}-107^{\circ}$. Although our $\theta$ is somewhat larger than the widely accepted value $\theta$ $=103^{\circ}-105^{\circ}$ for $l$-Se slightly above its melting point. By taking the $Q$ resolution into account, we conclude $\theta$ $=107^{\circ} \pm 5^{\circ}$.

We would like to point out that it would be possible to investigate finer structures if sufficiently good $Q$ resolution and intensity were achieved. For example, the detailed oscillation at low- $Q$ region in the constant- $E$ spectra in the experimental data in Fig. 2(b) implies long-range, probably intrachain, correlations for the stretching mode. However, the point about our analysis for $l$-Se is that the primary $Q$ dependence can be derived from a simple model with clearcut physical meaning of three-body correlations. There are several good reasons and past works which support our analysis. For example, the dihedral angle $\phi$, shown in Fig. 3(b), is known to have a much wider distribution than $r$ and $\theta$, as have been shown by $a b$ initio MD simulations. ${ }^{21,22}$ In fact, 
the detailed analysis of $S(Q)$ have shown that a free-rotatingchain model with constant $r$ and $\theta$, without any restrictions to $\phi$, is a good structural model for $l$-Se. ${ }^{23}$ The wide distribution of $\phi$ will smear out the oscillation due to the fourbody correlations in the $Q$ space. In addition, a study on the vibrational modes of regular helical Se chain have revealed that $\phi$ is not a dominant factor that affects the vibrations of Se systems. ${ }^{24}$ We have checked this analysis method can be also applied to the liquid $\mathrm{Te}_{50} \mathrm{Se}_{50}$ in its semiconducting region, where the structure is known to be chainlike. In its metallic region, the model could not be simply applied, due to the complexity of the structure.

In this Brief Report, we have measured $S(Q, E)$ by INS for $l$-Se at slightly above the melting point and analyzed the $Q$ dependence of the intensity of the bond-stretching mode. Since this is the mode that has the highest energy, the $Q$ dependence of its intensity is expected to be governed by rigid structures such as intramolecular short-ranged correlations. Therefore, we modeled the $Q$ dependence of $S(Q, E)$ using small cluster models that deal with two-body and three-body correlations. The most important results are that we could quantitatively extract the short-range structures from such $Q$ dependences, directly from the dynamics, and that the model can provide a basic and clearcut physical understanding of $Q$ dependences.

We thank F. J. Bermejo for valuable discussions in the initial stages of the analysis. This work was partially supported by a Grant-in-Aid for Scientific Research from MEXT, Japan, and was carried out under the UK-Japan collaboration on neutron scattering. One of the authors (A.C.) is supported by the JSPS for Research Abroad.
${ }^{1}$ J. Hafner, J. Phys. C 14, L287 (1981); 16, 5773 (1983); G. S. Grest, S. R. Nagel, and A. Rahman, Phys. Rev. Lett. 49, 1271 (1982).

${ }^{2}$ J. P. Hansen and I. R. McDonald, Theory of Simple Liquids (Academic, New York, 1986); for a review on the phonon wave propagation in molten salts, see S. Okazaki, Y. Miyamoto, and I. Okada, Phys. Rev. B 45, 2055 (1992).

${ }^{3}$ F. J. Bermejo, J. W. Taylor, S. E. McLain, I. Bustinduy, J. F. C. Turner, M. D. Ruiz-Martin, C. Cabrillo, and R. FernandezPerea, Phys. Rev. Lett. 96, 235501 (2006).

${ }^{4}$ J. M. Carpenter and D. L. Price, Phys. Rev. Lett. 54, 441 (1985); D. L. Price and J. M. Carpenter, J. Non-Cryst. Solids 92, 153 (1987).

${ }^{5}$ For, e.g., the analysis of $S(Q, E)$ by use of the dynamic radial distribution function, $g(r, E)$, is reviewed in R. J. McQueeney, Phys. Rev. B 57, 10560 (1998).

${ }^{6}$ F. J. Bermejo, M. Alvarez, S. M. Bennington, and R. Vallauri, Phys. Rev. E 51, 2250 (1995).

${ }^{7}$ F. J. Bermejo, R. Fernández-Perea, M. Alvarez, B. Roessli, H. E. Fischer, and J. Bossy, Phys. Rev. E 56, 3358 (1997); we note that opinions vary on the point that the mode is optic or acoustic. See also T. Scopigno, A. Filipponi, M. Krisch, G. Monaco, G. Ruocco, and F. Sette, Phys. Rev. Lett. 89, 255506 (2002).

${ }^{8}$ W.-C. Pilgrim, M. Ross, L. H. Yang, and F. Hensel, Phys. Rev. Lett. 78, 3685 (1997).

${ }^{9}$ J. E. Bove, F. Formisano, E. Guarini, A. Ivanov, C. Petrillo, and F. Sacchetti, Europhys. Lett. 79, 16002 (2007).

${ }^{10}$ T. Bryk and I. Mryglod, J. Phys.: Condens. Matter 14, L445 (2002).

${ }^{11}$ V. F. Sears, Neutron News 3, 26 (1992).
${ }^{12}$ For the assignments of the vibrational modes, see, e.g., F. Gampf, in The Physics of Selenium and Tellurium, edited by E. Gerlach, P. Grosse (Springer-Verlag, Berlin, 1979), p. 64; The Physics of Selenium and Tellurium, edited by W. C. Cooper (Pergamon, New York, 1969); S. N. Yannopoulos and K. S. Andrikopoulos, J. Chem. Phys. 121, 4747 (2004).

${ }^{13}$ A. Chiba, Y. Ohmasa, and M. Yao, J. Chem. Phys. 119, 9047 (2003), and references therein.

${ }^{14}$ J. M. Carpenter and C. A. Pelizzari, Phys. Rev. B 12, 2391 (1975); 12, 2397 (1975). See also J. M. Carpenter, J. Chem. Phys. 46, 465 (1967).

${ }^{15}$ See, e.g., W. A. Phillips, U. Buchenau, N. Nüucker, A. J. Dianoux, and W. Petry, Phys. Rev. Lett. 63, 2381 (1989).

${ }^{16}$ This is a result of the electronic configuration of Se. See, e.g., Ref. 21 or 12.

${ }^{17}$ A. Chiba, Y. Ohmasa, M. Yao, and S. M. Bennington, J. Neutron Res. 12, 301 (2004).

${ }^{18}$ K. Tamura and M. Inui, J. Phys.: Condens. Matter 13, R337 (2001).

${ }^{19}$ M. Edeling and W. Freyland, Ber. Bunsenges. Phys. Chem. 85, 1049 (1981).

${ }^{20} \mathrm{~K}$. Tamura and S. Hosokawa, Ber. Bunsenges. Phys. Chem. 96, 681 (1992).

${ }^{21}$ D. Hohl, and R. O. Jones, Phys. Rev. B 43, 3856 (1991).

${ }^{22}$ G. Kresse, F. Kirchhoff, and M. J. Gillan, Phys. Rev. B 59, 3501 (1999); F. Kirchhoff, G. Kresse, and M. J. Gillan, ibid. 57, 10482 (1998).

${ }^{23}$ M. Misawa and K. Suzuki, Trans. Jpn. Inst. Met. 18, 427 (1977); J. Phys. Soc. Jpn. 44, 1612 (1978).

${ }^{24}$ K. Nakamura and A. Ikawa, Phys. Rev. B 66, 024306 (2002). 